\title{
Unexpected Diagnosis
}

\author{
Gauravpal S. Gill ${ }^{1}$, Rukma R. Govindu ${ }^{2}$, Ragai Fouda ${ }^{3}$, Hussam M. Ammar ${ }^{1}$ \\ 1. Internal Medicine, Medstar Washington Hospital Center, Washington DC, USA 2. Internal Medicine, The University \\ of Texas Health Science Center at Houston, Houston, USA 3. Internal Medicine/cardiology, George Eliot Hospital Nhs \\ Trust, Nuneaton, GBR
}

Corresponding author: Hussam M. Ammar, h_ammar68@yahoo.com

\section{Abstract}

A 74-year-old man presented to the ER with an eight-month history of shortness of breath, cough, anorexia, and weight loss. He had emigrated from sub-Saharan African to the USA, where he was diagnosed and treated for coronary artery disease, heart failure, and stroke; was hospitalized several times; and underwent hernia surgery. Despite the complex care that he received in the USA for many years, the diagnosis of AIDS was continually missed for years, and the patient was eventually diagnosed at the age of 74 .

Categories: Internal Medicine, Medical Education, HIV/AIDS

Keywords: pneumocystis pneumonia, aids, hiv, limited english proficiency, diagnostic momentum, diagnostic parsimony, occam's razor, hicham's dictum.

\section{Introduction}

An estimated 1.1 million people in the USA live with HIV [1]. One in seven HIV-positive individuals is unaware of the infection, and $20 \%$ of individuals who are aware of their HIV diagnosis have not been linked to care [1-2]. Greater age is significantly associated with greater delays in diagnosis (median delay: 4.5 years in people aged $\geqslant 55$ years vs. 2.4 years in people aged $13-24$ years) [2]. Approximately $55 \%$ of adults in the USA have never been screened for HIV [3].This case report presents the journey of one of the 1.1 million patients living with HIV; this particular patient remained undiagnosed until the age of 74 .

\section{Case Presentation}

A 74-year-old man presented to the ER with an eight-month history of shortness of breath, coughing, anorexia, and weight loss without a fever. On examination, the patient was fully alert and oriented with a blood pressure of $80 / 60 \mathrm{mmHg}$, a regular pulse of 80 beats per minute, a respiratory rate of 18 breaths per minute, a temperature of $36.8^{\circ} \mathrm{C}$, and a body mass index of $19 \mathrm{~kg} / \mathrm{m} 2$. The jugular venous pulse pressure was elevated to $12 \mathrm{~cm} \mathrm{H2O}$, and bilateral fine basal crackles were audible on pulmonary auscultation. Cardiac examination revealed normal heart sounds and a holosystolic murmur with a grade of $3 / 6$ that was best heard over the apex, with no gallop. He did not have lymphadenopathy or hepatosplenomegaly. The patient exhibited bitemporal wasting and diffuse wasting of the arm and leg muscles. His extremities were warm, and his peripheral pulses were palpable. Our patient had been previously diagnosed with congestive heart

Received 07/29/2019

Review began 07/31/2019 Review ended 09/13/2019 Published 09/26/2019

(c) Copyright 2019 Gill et al. This is an open access article distributed under the terms of the Creative Commons Attribution License CC-BY 3.0., which permits unrestricted use, distribution, and reproduction in any medium, provided the original author and source are credited. failure, atrial fibrillation, left ventricular aneurysm with left ventricular thrombus, and stroke, and two coronary stents had been placed a few years prior. He could walk only a few steps before feeling shortness of breath; he was fatigued most of the time, and he had lost approximately $7.7 \mathrm{~kg}$ in the previous year. His home medications included clopidogrel, apixaban, lisinopril, carvedilol, and furosemide. At the time of presentation, his serum albumin level was $1.6 \mathrm{~g} / \mathrm{dL}$, and his hemoglobin level was $11 \mathrm{~g} / \mathrm{dL}$. Chest radiography revealed cephalization and alveolar and reticular opacities (Figure 1). 


\section{Cureus}

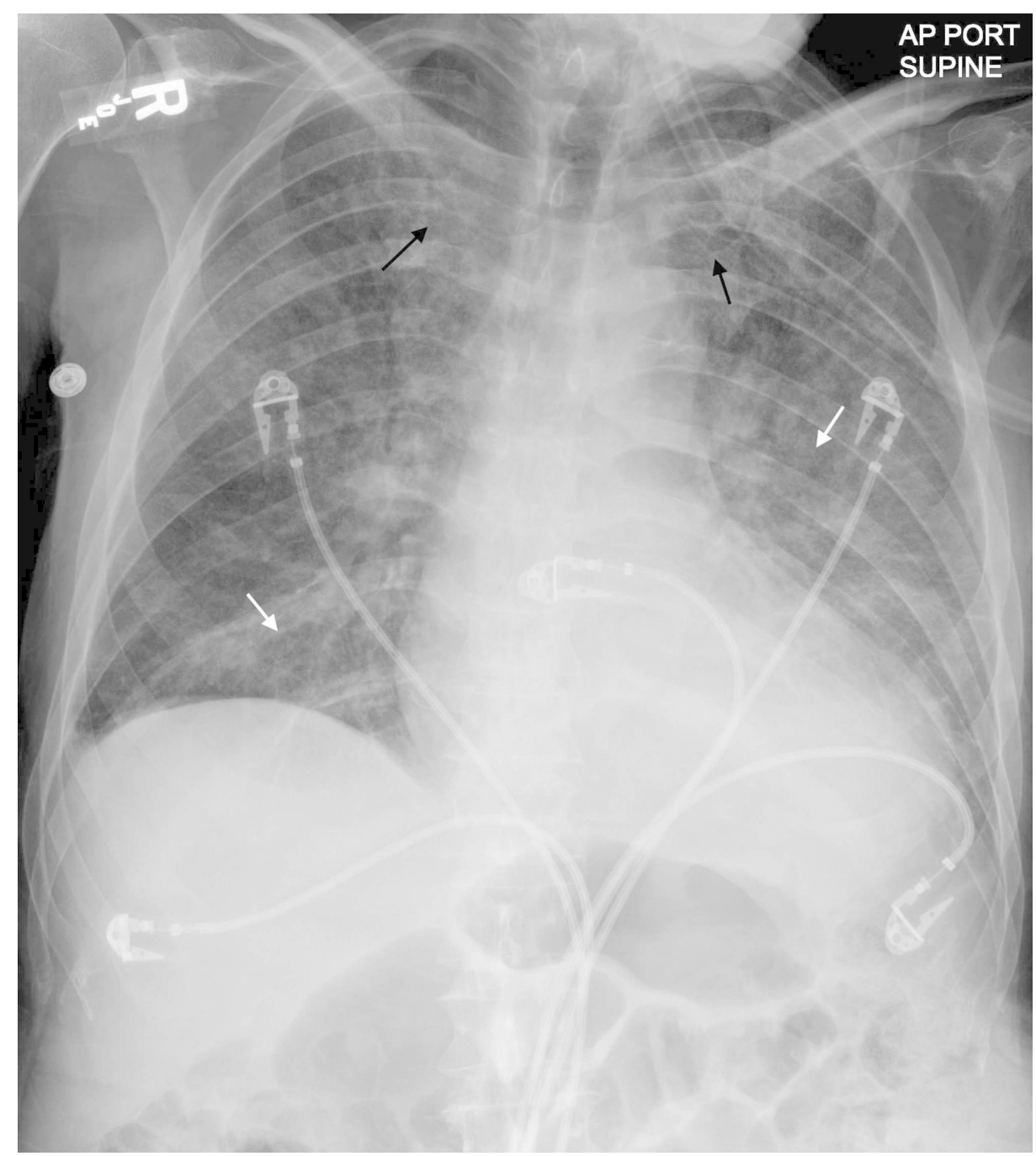

\section{FIGURE 1: Portable anteroposterior chest radiography.}

Cephalization (black arrows), alveolar and reticular opacities (white arrows).

The patient received $2 \mathrm{~L}$ of intravenous $0.9 \%$ sodium chloride in the ER. His blood pressure improved to $110 / 80 \mathrm{mmHg}$, and his creatinine level improved from 2.1 to $1.2 \mathrm{mg} / \mathrm{dL}$. The patient, a retired businessman living with his family in the Southeast United States, was originally from sub-Saharan Africa and had immigrated to the USA 10 years prior. He had traveled to his home country in sub-Saharan Africa twice a year for the past 5 years. He had been receiving medical care in the USA and had undergone hernia surgery and percutaneous coronary intervention a few years previously. He had also been hospitalized a few times for pneumonia and diarrhea.

We interviewed the patient over the phone with the aid of an interpreter. He remembered that he had received a tuberculin skin test a decade prior, which produced a negative result. He did not recall any contact with patients with tuberculosis (TB). He reported experiencing intermittent watery, nonbloody diarrhea for the past six months, and he had used over-the-counter diphenoxylate, which resulted in some improvement in his symptoms.

The observed hypotension, elevated jugular venous pulse pressure, and fine basal crackles suggested a diagnosis of heart failure, but the patient's warm extremities and normal mentation rendered a diagnosis of cardiogenic shock unlikely. We were careful not to fall into the traps of diagnostic momentum and assume that the clinical presentation was solely secondary to heart failure [4]. A few red flags emerged in this case. First, the patient was a foreign-born American citizen. The rate of TB is 13.4-fold higher among foreign-born persons in the USA than among US-born persons [5]. The language barrier prevented clear communication, which can sometimes lead physicians to incorrect diagnoses [6]. Although pneumonia and diarrhea diagnoses are not uncommon in a 74-year-old man, the patient's multiple hospital admissions without a definitive diagnosis or successful treatment suggested a possible missed diagnosis. Sub-Saharan Africa, where this man traveled from and had lived for most of his life, has the highest prevalence of HIV infection 


\section{Cureus}

in the world; this region is home to only $12 \%$ of the global population but accounts for $71 \%$ of the global burden of HIV infection [7]. The tuberculin test also has limitations. The test has a sensitivity of $71 \%-82 \%$ in patients with latent TB, but its sensitivity drops to only $43 \%$ in immunocompromised patients with HIV [8]. The findings on chest radiography could have been secondary to the known heart failure diagnosis of the patient, perhaps due to atypical pneumonia or fungal or viral infection. Pneumocystis pneumonia (PCP) can also cause a similar radiological pattern. He may also have had a combination of both heart failure and underlying infection.

The chest radiography findings did not rule out pulmonary TB; the incidence of radiographic findings that are not typical for primary or reactivated TB is $8 \%-29 \%$, and even normal chest radiography results are observed in $1 \%-9.2 \%$ of patients with culture-proven pulmonary TB and in $22 \%$ of HIV-positive patients with pulmonary TB [9-10].

The patient became hypoxic overnight, and chest radiography revealed interval progression with the development of florid pulmonary edema and extensive alveolar opacities. The electrocardiogram did not reveal new ST-T changes. Serial troponin tests were negative. Bilevel positive oxygen therapy was started, and $100 \mathrm{mg}$ of intravenous furosemide was administered over 12 hours. The patient's oxygenation improved by the end of the night (Figure 2).

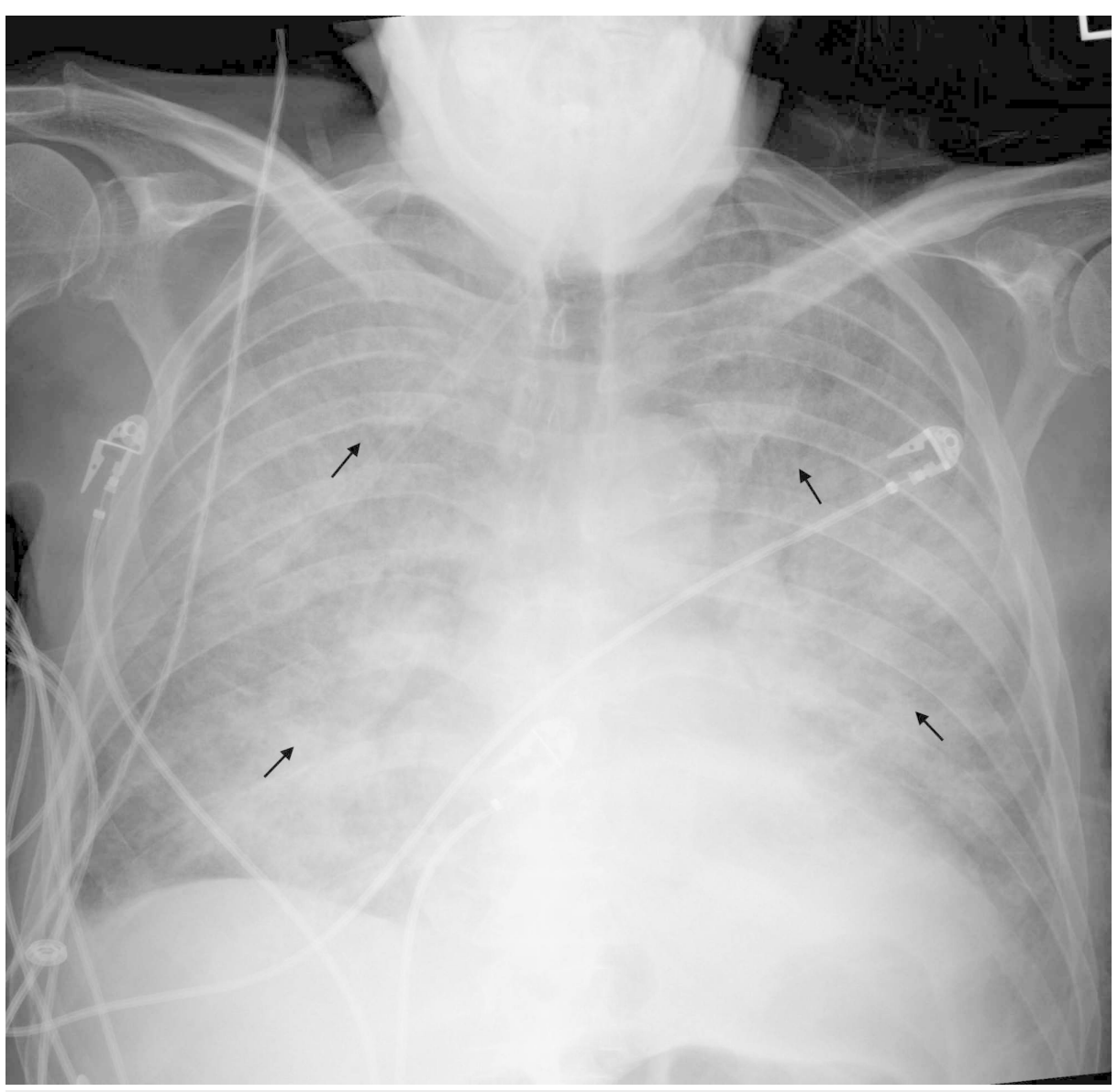

\section{FIGURE 2: Portable anteroposterior chest radiography.}

Interval progression with development of extensive bilateral alveolar opacities (black arrows).

A fourth-generation HIV test was positive, the CD4 count was 50 cells/ $\mu \mathrm{L}$, and the HIV viral load was 7,078,332 copies/mL. Three sputum samples tested negative for acid-fast bacilli by Ziehl-Neelsen staining and an Xpert MTB\RIF assay (Cepheid, Sunnyvale, CA, USA).

We examined the chest radiography results through the lens of HIV. PCP is the most common opportunistic infection in HIV patients and is a diagnosis that should not be missed in an AIDS patient with respiratory symptoms and a CD4 count of 50 cells/ $\mu \mathrm{L}$. Typical radiographic features include bilateral perihilar interstitial infiltrates, but chest radiographs are normal in $33 \%$ of cases [11]. Less common findings include solitary or multiple nodules, upper lobe infiltrates in patients receiving pentamidine, pneumatoceles, and pneumothorax [11]. Fever may be absent in $20 \%$ of cases. Cough is usually dry but can be productive in $30 \%$ 


\section{Cureus}

of cases, and shortness of breath occurs in $68 \%$ of cases [12]. The duration of symptoms extends over weeks to months. Chest CT revealed bilateral alveolar opacities, pneumatoceles, and small bilateral pleural effusion (Figure 3).

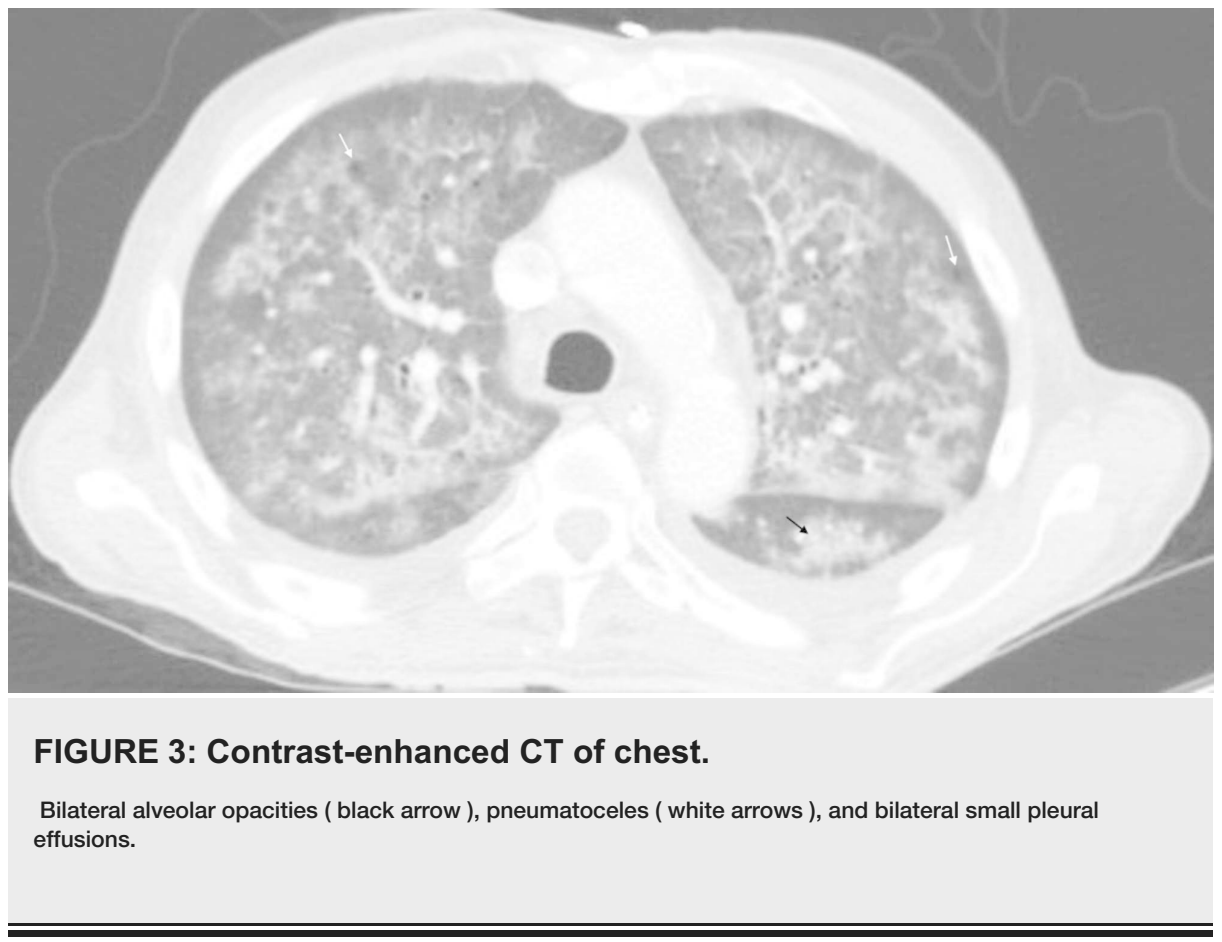

We initiated empiric treatment with intravenous trimethoprim-sulfamethoxazole. Corticosteroid therapy was not indicated based on the severity of the patient's illness. Urgent bronchoscopy and bronchoalveolar lavage (BAL) examination were performed and revealed PCP by Giemsa staining.

Our patient tolerated his treatment well and returned to living with his family in the Southeast United States. The TB sputum culture remained negative. We were last updated by the patient's family five months after his discharge that he had started antiretroviral therapy, was attending an outpatient rehabilitation center once a week, and was planning to visit his home country in Africa in several weeks.

\section{Discussion}

The patient in this case is one of the 1.1 million people in the USA who are living with HIV. In 2006, the Centers for Disease Control (CDC) recommended that adolescents and adults between the ages of 13 and 64 years should be screened for HIV infection and that the written informed consent requirement should be eliminated [13]. Our patient would not have been screened under the CDC guidelines. The state in which our patient lived did not eliminate the requirement for written informed consent for HIV testing until 2015 [14]. A routine opt-out HIV testing program in emergency departments would have probably captured his diagnosis during one of his several hospitalizations or ER visits if it had been in effect where he had received his medical care. Patients with limited English proficiency (LEP), such as our patient, have worse clinical outcomes than those with high proficiency and experience a higher incidence of medical errors resulting in physical harm $[6,15]$. One study found that professional interpreters are often not used for patients with LEP: only approximately $60 \%$ of patients reported the presence of any kind of interpreter with physicians during their hospitalizations [16].

Diagnostic parsimony is a concept that physicians commonly utilize in their clinical reasoning. As physicians, we have correctly been taught to first look for a single unifying diagnosis that explains different symptoms, signs, and test results in our patients rather than to look for two or three unrelated

diagnoses [17-18]. The concept of diagnostic parsimony is based on an ancient Greek philosophical principle that is summarized by the Latin phrase "entia non sunt multiplicanda praeter necessitate," which translates to "entities must not be multiplied beyond necessity." This principle is commonly known as Occam's razor, named for the 14th-century philosopher William of Ockham [18-19]. Heart failure was the simplest and most likely diagnosis for our patient, especially because the patient also had a known history of heart failure and had been hospitalized several times for the condition.

Fortunately, we made the judgement that it was best not to rely on this principle in our search for a diagnosis in this case. We instead applied Hickam's dictum, "patients can have as many diseases as they damn well please”, named after John Hickam, the Chair of Medicine at Indiana University (1914-1970). Ultimately, we arrived at three diagnoses: congestive heart failure, AIDS, and PCP [18-19]. The concept of 
diagnostic parsimony is not appropriate for immunocompromised patients, polypharmacy cases, and elderly patients, in whom disease presentations are atypical and having more than one diagnosis is not uncommon [17-18]. Diagnostic parsimony must be used with caution, and even arriving at an established diagnosis does not exclude the coexistence of others [20].

\section{Conclusions}

HIV often masquerades as other illnesses and should therefore be considered and screened for in clinical practice. Clinicians must be skeptical; they should not fall into the trap of diagnostic momentum or accept diagnostic labels at face value. Our healthcare system failed our patient for several years; however, thinking outside the box enabled us to make the correct diagnosis in only a few days.

\section{Additional Information \\ Disclosures}

Human subjects: Consent was obtained by all participants in this study. Conflicts of interest: In compliance with the ICMJE uniform disclosure form, all authors declare the following: Payment/services info: All authors have declared that no financial support was received from any organization for the submitted work. Financial relationships: All authors have declared that they have no financial relationships at present or within the previous three years with any organizations that might have an interest in the submitted work. Other relationships: All authors have declared that there are no other relationships or activities that could appear to have influenced the submitted work.

\section{Acknowledgements}

We would like to thank the patient presented in this case for allowing us to publish and share his story with readers around the world. We are also grateful to the staff of unit 4G at MedStar Washington Hospital Center for their outstanding performance that saved our patient's life.

\section{References}

1. U.S. Statistics. (2017). Accessed: September 10, 2018: https://www.hiv.gov/hiv-basics/overview/data-andtrends/statistics.

2. Dailey AF, Hoots BE, Hall HI, et al.: Vital signs: human immunodeficiency virus testing and diagnosis delays -United States. Morb Mortal Wkly Rep. 2017, 66:1300-1306. 10.15585/mmwr.mm6647e1

3. Rizza SA, MacGowan RJ, Purcell DW, Branson BM, Temesgen Z: HIV screening in the health care setting: status, barriers, and potential solutions. Mayo Clin Proc. 2012, 87:915-924. 10.1016/j.mayocp.2012.06.021

4. Croskerry P: Achieving quality in clinical decision making: cognitive strategies and detection of bias . Acad Emerg Med. 2002, 9:1184-1204. 10.1197/aemj.9.11.1184

5. Scott C, Kirking HL, Jeffries C, Price SF, Pratt R: Tuberculosis trends--United States, 2014. Morb Mortal Wkly Rep. 2015, 64:265-269.

6. Divi C, Koss RG, Schmaltz SP, Loeb JM: Language proficiency and adverse events in US hospitals: a pilot study. Int J Qual Health Care. 2007, 19:60-67. 10.1093/intqhc/mzl069

7. Kharsany AB, Karim QA: HIV infection and AIDS in Sub-Saharan Africa: current status, challenges and opportunities. Open AIDS J. 2016, 10:34-48. 10.2174/1874613601610010034

8. Lewinsohn DM, Leonard MK, LoBue PA, et al.: Official American Thoracic Society/Infectious Diseases Society of America/Centers for Disease Control and Prevention clinical practice guidelines: diagnosis of tuberculosis in adults and children. Clin Infect Dis. 2017, 64:111-115. 10.1093/cid/ciw778

9. Pepper T, Joseph P, Mwenya C, et al.: Normal chest radiography in pulmonary tuberculosis: implications for obtaining respiratory specimen cultures. Int J Tuberc Lung Dis. 2008, 12:397-403.

10. Cudahy P, Shenoi SV: Diagnostics for pulmonary tuberculosis. Postgrad Med J. 2016, 92:187-193. 10.1136/postgradmedj-2015-133278

11. Kennedy CA, Goetz MB: Atypical roentgenographic manifestations of pneumocystiscariniipneumonia . Arch Intern Med. 1992, 152:1390-1398. 10.1001/archinte.1992.00400190032008

12. Kovacs JA, Hiemenz JW, Macher AM, et al.: Pneumocystis carinii pneumonia: a comparison between patients with the acquired immunodeficiency syndrome and patients with other immunodeficiencies. Ann Intern Med. 1984, 100:663-671. 10.7326/0003-4819-100-5-663

13. Branson BM, Handsfield HH, Lampe MA, Janssen RS, Taylor AW, Lyss SB, Clark JE: Revised recommendations for HIV testing of adults, adolescents, and pregnant women in health-care settings. MMWR Recomm Rep. 2006, 55:1-17.

14. The Florida Senate. CS/CS/HB 321: HIV testing. (2015). Accessed: September 5, 2018: https://www.flsenate.gov/Session/Bill/2015/321/Analyses/h0321c.HHSC.PDF.

15. Flores G: Language barriers to health care in the United States . N Engl J Med. 2006, 355:229-231. 10.1056/NEJMp058316

16. Schenker Y, Perez-Stable EJ, Nickleach D, Karliner LS: Patterns of interpreter use for hospitalized patients with limited English proficiency. J Gen Intern Med. 2011, 26:712-717. 10.1007/s11606-010-1619-Z

17. Mani N, Slevin N, Hudson A: What three wise men have to say about diagnosis . BMJ. 2011, 343:7769. 10.1136/bmj.d7769

18. Hilliard AA, Weinberger SE, Tierney LM Jr, Midthun DE, Saint S: Clinical problem-solving. Occam's razor versus Saint's triad. N Engl J Med. 2004, 350:599-603. 10.1056/NEJMcps031794

19. McIntosh HD: Memorial. John Bamber Hickam, M.D. Trans Am Clin Climatol Assoc. 1971, 82:lx-lxii .

20. Schattner A: Caveats to the application of diagnostic parsimony-reply . QJM. 2016, 109:144. 


\section{Cureus}

10.1093/qjmed/hcv161 